\title{
MODELO DE DESARROLLO SOSTENIBLE REGIONAL Y EL PROGRAMA 21
}

\author{
Alfonso Romero B. ${ }^{*}$ y Jorge Inche M.*
}

\section{RESUMEN}

El presente articulo propone la hipótesis de desarrollar a las regiones en un mediano plazo si se hace una gestión optima de los gobiernos regionales, enmarcado en el modelo del desarrollo sostenible y las directivas del programa 21. Se destacan las fortalezas, debilidades y recomendaciones generales para cada aspecto del modelo de desarrollo sostenible regional.

Palabras clave: Desarrollo regional sostenible. Valorización de los recursos naturales. Patrimonio económico regional.

\section{ABSTRACT}

The present article proposes a hypothesis for developing regions on a short term by means of performing an optimal management of regional governments, framed within a sustainable development model as well as Program 21 guidelines. Strengths, weaknesses and general suggestions for each aspect of the regional sustainable development model are highlighted.

Key words: Sustainable regional development. Natural resources appraisal. Regional economic patrimony.

\section{INTRODUCCIÓN}

En el Perú, la implementación de los Gobiernos Regionales tiene un panorama poco alentador, pues las última vez que se intentó en la década de los 80 fue un fracaso total, esto conlleva a tener mucho profesionalismo y menos demagogia en los siguientes meses que cambiará totalmente la organización del País, abriendo la posibilidad de sacarlo del subdesarrollo en un corto plazo. En lo concerniente al desarrollo sostenible algunas Instituciones como el Instituto Nacional de Recursos Naturales (INRENA), Instituto del Mar Peruano (IMARPE), Consejo Nacional del Ambiente (CONAM), y las Direcciones de Asuntos Ambientales de los Ministerios deben propiciar una adecuada política de participación en la nueva organización del País.

Actualmente, los gobiernos y la población en general en todo el mundo luchan contra el uso y explotación desmedida de los recursos naturales, la erosión cos-

" Instituto de Investigación de la Facultad de Ingeniería Industrial.

UNMSM

E-mail: iifi@unmsm.edu.pe tera, los derrames de petróleo y la contaminación del agua potable, en tanto que, la posibilidad de desarrollo de las regiones así como la mejora en la calidad de vida de los habitantes dependen del uso óptimo de sus recursos naturales y de su medio circundante, pues como se sabe, las cuestiones como el crecimiento de la población, la deforestación, la lluvia ácida y la posibilidad de rápidos cambios climáticos son otras variables de decisiones difíciles para el futuro.

En 1975, un grupo internacional de estudio reunido en Belgrado, Yugoslavia, propuso un marco de referencia mundial para la educación ambiental, el cual se llamó la "Carta de Belgrado». Esta Carta afirma que la meta de la educación ambiental es hacer que la población mundial se preocupe por el medio ambiente y tenga el conocimiento y la dedicación que la capacite para buscar soluciones a los problemas actuales y prevenir problemas nuevos.

Una sociedad sostenible, que se define como la que satisface sus necesidades sin disminuir los recursos que requieren las generaciones futuras, ha llegado a ser meta suprema de la educación ambiental. 


\section{FUNDAMENTO DEL MODELO DE DESARROLLO SOSTENIBLE}

En Diciembre del 2000, la Asamblea General de las Naciones Unidas (ONU) acordó realizar la cumbre mundial de desarrollo sostenible en Sudáfrica, Johannesburgo, con la finalidad de reinsertar el concepto de "desarrollo sostenible" dentro de la agenda internacional de los gobiernos del mundo.

El modelo posee el sustento de aplicación de los documentos existentes y discutidos por los expertos del mundo en las distintas sesiones mundiales de protocolo, donde se trazan lineamientos generales para la mejora de la calidad de vida en el planeta Tierra, entre ellos tenemos los acuerdos de Río de Janeiro 1992, Acuerdo de Santa Cruz, 1999, Acuerdo de Huaraz, Julio del 2002 y el reciente acuerdo de Johannesburgo, agosto del 2002.

\section{COMPONENTES DEL MODELO DE DESARROLLO}

\section{La Agenda 21}

Es un documento estratégico que fue aprobada en la conferencia de las Naciones Unidas para el Medio Ambiente y para el Desarrollo que se celebró en Río de Janeiro el año 1992, donde estuvieron 179 Estados, que diseñaron el prototipo de los modelos de desarrollo sostenible. Este documento constituye un plan de acción para la década de los 90 y viene a ser la alianza global de la humanidad para el medio ambiente y el desarrollo. Es un documento extenso estructurado en 40 capítulos.

\section{Definición de necesidades}

- Diseño y funcionamiento de un sistema de información regional de desarrollo sostenible.

- Organización de los sectores de la sociedad para la educación en sostenibilidad.

- Elaboración del marco conceptual.

- Preparación de la ejecución.

Diseño de pilotaje y análisis para puesta en marcha en corto plazo

- Discretización de la zona geográfica en estudio.

- Diseño del muestreo, método y técnica.

- Toma de muestras de diversos medios.

- Análisis estadístico de datos.

Contrastación de los niveles empíricos disponibles con niveles de referencia

- Tablas de niveles de referencia y su comparación con datos analizados (EPA, SSL, RBCA).
- Niveles genéricos y vía de migración.

- Expresiones de cálculo.

- Niveles de aproximación.

- Contraste de valores empíricos y calculados.

- Análisis de riesgo.

Definición de las acciones posteriores

- Apertura de temas de indicadores de control real y dinámico.

- Manejo de resultados de la cuantificación de la valoración económica

- Importancia del aporte científico o social en la gestión regional.

- Evaluación del Impacto socio económico y parámetros de control.

\section{Esquema de operación inicial}

- Morfología.

- Geología y estructuras.

- El medio físico.

- Inventario de recursos naturales.

- Valorización de los recursos naturales.

- Patrimonio Regional.

- Tesoro Público Regional.

\section{METODOLOGÍA DE DESARROLLO}

La implantación de este modelo se realizará mediante la conformación de equipos de trabajo a escala horizontal de ejecución de acciones así como la toma de decisiones, estos grupos organizados y seleccionados por equipos representativos, de los sectores pilares de desarrollo sostenible en cada Región es la que el modelo considerará como una variable importante de sostenibilidad, sin embargo en el presente trabajo se ha ensayado con el método del Focus Group ("Grupos focalizados en un tema»), en la Universidad Nacional Mayor de San Marcos. Concluyéndose que para la aplicación del modelo se conformarán equipos por cada variable del modelo y liderado por un responsable a tiempo completo. Este equipo de trabajo deberá presentar su presupuesto, estrategias de trabajo, cronograma y metodología de desarrollo de cada variable del modelo, el mismo que se simula con el aspecto fundamental de los principales sectores de sostenibilidad en la Región.

\section{CATASTRO DE ORDENAMIENTO TERRITORIAL Y URBANO}

Es una de las variables de la sostenibilidad regional porque permitirá modelizar digitalmente los recursos naturales, sociales y productivos de la Región, para esto se adquiere una imagen de satélite 
- una base de Datos Gis digital para la zona geográfica, estos materiales se pueden solicitar a costo muy bajo en el Instituto Geológico, Minero y Metalúrgico (INGEMMET) o el Ministerio de Energía y Minas (MEM). A esta base de datos se añade información específica de la zona geográfica a determinar como: Fuentes de contaminación, datos de calidad ambiental y zonas de alteración de elementos básicos del medio, recursos económicos productivos en agricultura, metales y no metales, hidrología, pesquería, energía y otros.

La aplicación de este modelo, permitirá plantear algunos planes regionales estratégicos de desarroIlo, anteproyectos de ley y la propuesta de creación de nuevas entidades rectoras de control y estandarización de las diversas actividades prioritarias que acelerarán el despegue de la región hacia una economía sostenida similar a los estados federados de los países desarrollados, cuyas potencialidades se reflejarán en:

- Plan Regional de educación ambiental, hacia el logro de la cultura ambiental.

- Plan Regional de investigaciones, UniversidadEmpresa.

- Plan Regional de turismo sostenible.

- Plan Regional de creación de incubadoras de microempresas.

- Plan Regional de inventario y valorización de recursos naturales.

- Plan Regional de energía.

- Plan Regional de liderazgo e identidad regional.

- Plan Regional de desarrollo sostenible.

Asimismo, entre las mesas de trabajo que se deben implementar son:
- Comunidades científicas y tecnológicas: Investigación y desarrollo.

- Ordenamiento y planificación del territorio: Inventario y valorización económica de recursos naturales.

- Fomento al desarrollo sostenible de los recursos humanos: Liderazgo e identidad.

- Comercio e industria: Incubadoras de microempresas.

- Mujer y desarrollo sostenible: Lucha contra la pobreza.

- Educación y cultura ambiental.

- Conservación de la diversidad biológica: Protección y fomento de la salud humana.

\section{CRONOGRAMA DE ACTIVIDADES}

El periodo de las actividades está determinado por los gobiernos regionales y/o locales, según como indica en el cuadro 1.

\section{ANÁLISIS DEL MODELO DE DESARROLLO SOSTENIBLE REGIONAL}

Para analizar los factores que determinan el modelo de desarrollo sostenible regional, se han establecido sesiones técnicas de Focus Group en la UNMSM, en el Instituto de Investigación de la Facultad de Geología, Minas, Metalurgia y Ciencias Geográficas, Instituto de Investigación de la Facultad de Ingeniería Industrial, Instituto de Investigación de Ciencias Sociales, Instituto de Investigación de Ingeniería Química; cuyos resultados son:

Cuadro 1. Cronograma de actividades.

\begin{tabular}{|c|c|c|c|c|c|c|c|c|}
\hline \multirow{2}{*}{ ACTIVIDADES } & \multicolumn{8}{|c|}{ MESES } \\
\hline & 1 & 2 & 3 & 4 & 5 & 6 & 7 & 8 \\
\hline $\begin{array}{l}\text { Reforzamiento } \\
\text { técnico del equipo } \\
\text { de trabajo }\end{array}$ & & & & & & & & \\
\hline $\begin{array}{l}\text { Sesiones de } \\
\text { metodología de } \\
\text { trabajo }\end{array}$ & & & & & & & & \\
\hline $\begin{array}{l}\text { Diseño y } \\
\text { preparación de } \\
\text { técnicas de } \\
\text { modelos }\end{array}$ & & & & & & & & \\
\hline $\begin{array}{l}\text { Discusión e } \\
\text { identificación } \\
\text { factores intrínsecos } \\
\text { de modelos de } \\
\text { desarrollo }\end{array}$ & & & & & & & & \\
\hline $\begin{array}{l}\text { Diseño e } \\
\text { identificación de } \\
\text { necesidad de datos }\end{array}$ & & & & & & & & \\
\hline Trabajo de campo & & & & & & & & \\
\hline Otros & & & & & & & & \\
\hline
\end{tabular}


Transferencia de tecnología en el desarrollo sostenible

Elaborar un diagnóstico de la participación de la Comunidad Científica y Tecnológica en el desarrollo de los proyectos en general con énfasis en temas ambientales nacionales, seguridad, calidad e impacto socio-económico.

Evaluar la transferencia tecnológica que se recibió durante la década de 1992-2002, por las distintas instituciones públicas y/o privadas así como los organismos $\sin$ fines de lucro en el Perú.

\section{a. Fortalezas}

- Existe mucho interés por la investigación de proyectos ambientales.

- Grandes oportunidades de participar en los talleres participativos para elaborar proyectos.

- Esfuerzo para publicar los resultados de trabajos de investigación, talleres, etc.

- Interés de la instituciones por intercambiar información vía correo electrónico.

- Esfuerzo para que se logre una mayor cobertura de convocatoria a los actores sociales.

b. Debilidades

- Problemas económicos para el financiamiento de proyectos sostenibles.

- Falta de información entre Instituciones.

- Falta de capacitación en temas ambientales actuales.

- Falta vinculación Universidad-Empresa-Estado.

- Carencia de un organismo que ordene y priorice las necesidades de investigación en el país.

c. Recomendaciones generales

- Mayor comunicación e integración entre la comunidad científica y la sociedad civil.

- Plantear una alianza estratégica y ponerse a trabajar en proyectos.

- En colegios, los temas de medio ambiente deben incluirse en todos los cursos como contenidos transversales.

- Que la educación ambiental se brinde en todos los niveles, sean colegios, comunidades, asociaciones, instituciones, universidades, etc.

Es necesario mencionar que, algunas Instituciones han venido realizando actividades en este campo, las que se detallan a continuación:

1. Facultad de Ingenieria Ambiental. Universidad Nacional del Callao

"Recuperación de los humedales de Ventanilla"

La Facultad de Ingeniería Ambiental se preocupa en mejorar la belleza paisajística del distrito, para ello viene trabajando en la recuperación del equilibrio ecológico de los humedales de Ventanilla.

En la primera visita a los humedales se pudo observar una excesiva cantidad de residuos sólidos en la zona, desorden en el crecimiento de la flora, contaminación de las aguas del pantano, invasión de la población, falta de autoridades competentes para el resguardo de la zona.

De acuerdo a lo observado se vienen realizando las siguientes actividades:

- Clasificación de los residuos sólidos de acuerdo a su toxicidad.

- Campaña de limpieza de la zona con participación de toda la facultad.

- Constantes visitas para el ordenamiento de la zona y concientizar a los pobladores.

- Muestreo de agua, flora y fauna.

- Muestreo microbiológico del agua.

- Análisis de calidad de agua.

- Inventario de flora y fauna.

- Búsqueda de legislación para declarar a los humedales de Ventanilla como un área ecológica.

2. Instituto de Investigación de la Facultad de Geología, Minas, Metalurgia y Ciencias Geográficas. UNMSM. Grupo de Investigación Geográfica Ambiental.

"Plan de Desarrollo Urbano Participativo del Distrito de Comas" $y$ «Red de Información General para la Gestión Urbana”

El área geográfica es el distrito de Comas se encuentra ubicado en el Cono Norte de Lima Metropolitana, en la región natural de la costa, provincia y departamento de Lima. La superficie total del distrito es de 4,875 hectáreas $\left(48.72 \mathrm{Km}^{2}\right)$ lo que representa el $5 \%$ del territorio del Cono Norte y $1.7 \%$ de Lima Metropolitana. Asimismo, los beneficiados de este proyecto son 457000 habitantes.

El Plan de Desarrollo Urbano Participativo viene a ser el primer plan urbano desarrollado íntegramente en tres niveles, el técnico, el nivel políti$\mathrm{co}$, y el nivel participativo. Esta último nivel se desarrollo a través de talleres por líneas estratégicas, áreas territoriales y distrital.

Para efectos de este proyecto la participación se basa en desarrollar investigación en las líneas de: Salud, saneamiento básico, contaminación ambiental (agua, aire, suelo), desorden social, recursos naturales análisis físico territorial del distrito de Comas. Para este proyecto se contó con el aporte científico y tecnológico del Instituto de Investigación de Facultad de Geo- 
logía, Minas, Metalurgia y Geográfica, docentes, egresados y alumnos.

Se estableció las siguientes propuestas de solución:

- Establecer Mesas de trabajo entre equipos multidisciplinarios (Municipio-USE- Universidades-Empresas, etc.)

- Recopilación de la información sobre proyectos ambientales.

- Asesorar a las comunidades sobre el medio ambiente por medio de un organismo facilitador.

- Plantear una legislación de transferencia tecnológica para fines ambientales.

- Identificaciones de líderes distritales, con brigadistas ecológicos para el monitoreo, supervisión capacitación y movilización.

3. Instituto de Investigación de la Facultad de Ingeniería Industrial. UNMSM

"Manejo de Residuos Sólidos mediante Segregación en la Fuente en Lima Cercado"

El estudio presenta una metodología para el manejo de los residuos sólidos a nivel de plan piloto en una zona de 2000 predios del distrito de el Cercado en Lima. Esta propuesta forma parte de la política de manejo de residuos sólidos de la municipalidad de Lima.

El estudio comprende 7 fases: Implementación de los recursos, estudio de la población, diseño de la campaña de sensibilización, ejecución de la sensibilización, análisis de resultados, propuesta del plan final y puesta en marcha.

El estudio brinda lecciones a seguir para instituciones que deseen involucrase en el tema de la sensibilización ciudadana y los aspectos básicos para la gestión de residuos. Los datos presentados pueden ser tomados para cálculos económicos y de dimensionamiento en proyectos similares ya que son fruto del trabajo de campo.

\section{Mujer y desarrollo sostenible}

La participación de la mujer se sustenta en:

- Los cambios necesarios para eliminar los obstáculos constitucionales, jurídicos, administrativos, culturales, sociales, económicos.

- La evaluación, revisión y aplicación de programas de enseñanza y materiales didácticos, a fin de fomentar entre hombres y mujeres la difusión de los conocimientos relacionados con la diferencia entre los sexos y la valoración de las funciones que puede realizar la mujer por medio de la enseñanza académica y no académica.
- La posibilidad de adoptar, reforzar y hacer cumplir una legislación que prohíba la violencia contra la mujer.

a. Fortalezas

- Apoyo la mujer por parte de las instituciones (ONGs, municipalidades, etc.) para que ella se desarrolle intelectualmente, con la finalidad de que pueda ocupar cargos institucionales, o dirigir su comunidad.

- Algunas instituciones realizan charlas con el objetivo de desarrollar el autoestima de la mujer.

\section{b. Debilidades}

- La mayoría de las mujeres no tienen el conocimiento necesario para ser autosuficiente.

- Falta de educación ambiental en la mujer.

- Ciertas instituciones brindan ayuda a la mujer mediante acciones paternalistas o semidependientes.

c. Recomendaciones generales

- Campañas para valorar a la mujer, a través de los medios, instituciones, comunidades (caminatas, pancartas, charlas, etc.).

- Presentar propuestas legales al ministerio de la mujer, para que mediante esta institución se pueda lograr constitucionalmente en el congreso la igualdad de derechos.

- Capacitar a las mujeres en el desarrollo de estrategias del cuidado del medio ambiente.

\section{Lucha contra la pobreza}

La lucha contra la pobreza se sustenta en:

- La subsistencia sostenible como un factor de integración.

- Lograr que todas las personas reciban, con carácter de urgencia, la oportunidad de trabajar.

- La elaboración de estrategias y programas para la reducción de la pobreza y sus efectos.

- La creación en los planes de desarrollo y los presupuestos nacionales, un núcleo de inversiones en capital humano, con políticas y programas especiales para las zonas rurales, los pobres de las zonas urbanas, la mujer y el niño.

a. Fortalezas

- El Perú cuenta con una megadiversidad de recursos naturales.

- Disponibilidad de técnicas de siembra, riego hidráulica etc, que nos dejaron los antepasados.

- Existencia de organizaciones camo (vasos de leche, comedores populares, etc.) que luchan contra la pobreza.

- El Estado está comenzando la descentralización. 
b. Debilidades

- Las leyes sobre conservación de recursos no se cumplen.

- La centralización no permite el desarrollo integral del país.

- Existencia de poblaciones que no cuentan con los servicios básicos.

- La inversión extranjera es mínima debido al sistema económico.

- No existe un plan de uso y aprovechamiento sostenible de los recursos naturales.

c. Recomendaciones generales

- Descentralización del poder económico y político creando en todo el país polos de desarrollo.

- Necesidad de una educación integral con mayores recursos económicos para desarrollar programas de educación adaptados a cada realidad.

- Identificar el rango de pobreza por la esperanza de vida, nivel educativo y ingreso per cápita.

- Definir y mejorar las relacionas entre la educación y las empresas privadas.

- El Estado debe de tener mayor control con la información de cada recurso natural para evitar la patentización de nuestros recursos por otros países.

- Cambiar las currículas de educación en todos los niveles, desarrollando planes integrales de acuerdo a su realidad y los avances de la tecnología.

\section{Población indígena y sus comunidades}

Al prestar su plena cooperación a las poblaciones indígenas y a sus comunidades, los gobiernos $y$, según procediera, las organizaciones intergubernamentales deberían proponerse el cumplimiento de lo siguiente:

1. Instituir un proceso encaminado a investir de autoridad a las poblaciones indígenas y a sus comunidades, mediante la adopción de medidas que incluyan:

- La adopción o ratificación de las políticas o instrumentos jurídicos apropiados a nivel nacional;

- El reconocimiento de que las tierras de las poblaciones indígenas y sus comunidades deben estar protegidas contra actividades que presenten riesgos para el medio ambiente o que la población indígena de que se trate considere improcedentes desde los puntos de vista social y cultural;

- El reconocimiento de sus valores, sus conocimientos tradicionales y sus prácticas de ordenación de los recursos, con miras a promover un desarrollo ecológicamente racional y sostenible;
- El reconocimiento de que la dependencia tradicional y directa de los recursos renovables y los ecosistemas, incluido el aprovechamiento sostenible, sigue siendo esencial para el bienestar cultural, económico y físico de las poblaciones indígenas y de sus comunidades;

- La elaboración y consolidación de los acuerdos nacionales sobre solución de controversias en lo que respecta al arreglo de problemas relacionados con la ordenación de tierras y la gestión de los recursos;

- La promoción de otros medios de producción ecológicamente racionales para asegurar diversas opciones respecto de como mejorar la calidad de su vida, de manera que puedan participar efectivamente en el desarrollo sostenible;

- La atención al aumento de la capacidad en favor de las comunidades indígenas, sobre la base de la adaptación y el intercambio de experiencias, conocimientos y prácticas de ordenación de los recursos tradicionales, para asegurar el desarrollo sostenible de esas comunidades;

2. Establecer, cuando proceda, acuerdos para intensificar la participación activa de las poblaciones indígenas y sus comunidades en la formulación de políticas, leyes y programas relacionados con la ordenación de los recursos en el plano nacional y otros procesos que pudieran afectarles, así como para propiciar que formulen propuestas en favor de políticas y programas de esa índole;

3. Participación de las poblaciones indígenas y sus comunidades, a los niveles nacional y local, en las estrategias de ordenación y conservación de los recursos y en otros programas pertinentes establecidos para apoyar y examinar estrategias de desarrollo sostenible, como las que se sugieren en otras áreas del programas 21.

a. Fortalezas

- Las poblaciones indígenas se sienten muy identificados culturalmente con su pasado y tratan de conservarlo.

- Poseen un espíritu comunal para trabajar en comunión con sus vecinos, además demuestran tener solidaridad entre ellos.

- Se puede apreciar que la unidad familiar es primordial entre ellos, no existiendo muchas casos de separaciones.

- Mantienen una convivencia equilibrada con el medio natural, preservando la biodiversidad.

- Poseen un vasto patrimonio cultural y se encargan de mantener vivas sus costumbres (fiestas patronales, comidas típicas, etc.), además practican la cosmovisión que se caracteriza por ser parte espiritual de las comunidades (rituales a la madre naturaleza, ritos para curaciones, etc.). 
- Su justicia se basa en el derecho consuetudinario, esto es, viven conforme a sus normas y se encargan de respetarlas y hacerlas cumplir.

- Se caracterizan por ser multilinguistas.

- Tienen un apreciado patrimonio comunal, que les permite trabajar sus tierras en comunidad.

- Existe gran diversidad de plantas medicinales que son de gran uso para curar o aliviar enfermedades.

b. Debilidades

- Pérdida progresiva de las lenguas nativas.

- El bajo nivel educativo, el pobre acceso a los servicios básicos y el avanzado empobrecimiento económico, son sólo algunas de las más importantes causas que generan la migración de las comunidades indígenas.

- La insuficiencia de vías de comunicación que nos permite a las comunidades el intercambio de sus productos, culturas, etc.

- La migración es uno de los problemas que afecta a la preservación de las comunidades, pero a su vez está condicionada de varias causas como el bajo nivel educativo, pobre acceso a los servicios básicos, empobrecimiento económico, etc.

c. Recomendaciones generales

- Promover una educación bilingüe intercultural, que mantenga la lengua nativa de la comunidad además del castellano.

- Descentralizar del poder político, económico y social otorgando a las comunidades las facultades de aprovechar racionalmente sus recursos naturales.

- Plantear una reforma legal y asesorar a las comunidades en el conocimiento de la normatividad existente y el respeto a las leyes por medio de un organismo encargado en este asunto.

- Formar líderes indígenas con convicción en sus ideales y con capacidad de llevar adelante su comunidad.

Fomento al desarrollo sostenible de recursos humanos

El objetivo general de los asentamientos humanos es mejorar la calidad social, económica y las condiciones de vida y de trabajo de todos, especialmente de los pobres de las zonas urbanas y rurales.

Ese mejoramiento deberá basarse en actividades de cooperación técnica, en la cooperación entre los sectores público, privado y de la comunidad, y en la participación de los grupos de la comunidad y los grupos de intereses especiales, como las mujeres, las poblaciones indígenas, los ancianos y los incapacitados.

a. Fortalezas

- Interés por parte del Estado en buscar apoyo económico (donaciones, prestamos) para el mejoramiento de la calidad de vida.
- La población asume un rol protagonista en procura de satisfacer sus necesidades básicas para su desarrollo.

- El Estado promueve la construcción de viviendas destinadas a la población.

b. Debilidades

- Deficiencia de las necesidades humanas educación, salud y vivienda. El $70 \%$ de la población no cuenta con viviendas adecuadas.

- Crecimiento desordenado de la población, no hay planificación por parte de las autoridades encargadas.

- Existe poco debate en torno a la calidad humana, el cual genera desmotivación y disminución del autoestima.

- Falta de un plan nacional de riesgos naturales, así como programas de reforestación, reciclaje, etc.

- Negligencia por parte de la población al ocupar zonas que no son aptas para vivienda, siendo estas vulnerables a los desastres naturales.

c. Recomendaciones generales

- Promocionar la construcción de viviendas que cuenten con los servicios básicos.

- Fortalecer a las Instituciones, empresas ONGs, organizaciones campesinas, que promuevan el desarrollo sostenible.

- Creación de proyectos que favorezcan al ordenamiento vehicular y territorial.

- Establecer medidas de reducción de daños causados por los desastres naturales, tales como: reubicación de la población, cercos naturales y construcción de centros de salud y rescate para los damnificados.

\section{La dimensión ambiental en la toma de decisiones}

Consiste en mejorar o reestructurar el proceso de adopción de decisiones, de manera que se integre plenamente en el examen de las cuestiones socioeconómicas y relativas al medio ambiente y se logre una participación más amplia del público. Sobre la base de que los países determinarán sus propias prioridades de conformidad con sus condiciones, necesidades, planes, políticas y programas nacionales, se proponen los objetivos siguientes:

- Fortalecer las estructuras institucionales a fin de permitir la plena integración de las cuestiones del medio ambiente y el desarrollo en todos los niveles del proceso de adopción de decisiones.

- Crear o mejorar mecanismos para facilitar la participación de organizaciones, grupos y particulares interesados en todos los niveles del proceso de adopción de decisiones. 
a. Fortalezas

- Capacidad humana de la población.

- Capacidad técnica de las universidades.

- Existencia de organismos con normas de saneamiento ambiental (las normas están diagnosticadas).

b. Debilidades

- Las instituciones ligadas al asunto ambiental no tinen niveles de decisión.

- Población con escasa asistencia técnica.

- Desorganización en las instituciones para la toma de decisiones.

- El bajo sitial que ocupa el medio ambiente para la toma de decisiones, siendo éste un factor primordial.

c. Recomendaciones generales

- Motivar la creación de brigadas ecológicas que promuevan la conciencia ambiental, y muestren la belleza de la fauna y flora y la relación directa que tiene el ambiente con el desarrollo humano.

- Presionar a las instancias pertinentes para hacer respetar las leyes y normas que rigen el cuidado y protección del medio ambiente.

- Capacitar a los líderes distritales en asuntos ambientales para trasmitir las ideas a las comunidades.

\section{Conservación de la diversidad biológica}

La diversidad biológica se sustenta en la utilización sostenible de los recursos biológicos, el suministro de los recursos genéticos y los informes mundiales sobre la biodicersidad.

a. Fortalezas

- La existencia de grupos multidisciplinarios que cada vez van creciendo con un solo interés, la investigación de proyectos ambientales.

- El correo electrónico se ha convertido en el nexo entre instituciones y entidades para intercambio de información más fluido y dinámico.

b. Debilidades

- Existencia de corrupción en las instancias encargadas de normar las legislaciones y hacerlas cumplir.

- Falta de interés común por lograr un cambio, erradicación de los procesos dañinos y hacer proyectos ambientales.

- Los obstáculos administrativos para crear vínculos con el exterior así como la falta de apoyo económico por parte del Estado o de la entidad privada para el financiamiento conjunto.

- Incumplimiento de las leyes que rigen a favor a la conservación de los recursos naturales. c. Recomendaciones generales

- Hacer estudios y conocer el potencial de recursos existentes, que ciertamente que se desconocen para su valoración.

- Proponer cambios en la legislación para la protección de la biodiversidad.

- Establecer un control de las especies nativas que se comercializan o exhiben en los países foráneos.

- Sancionar a los comercializadores de especies en vía de extinción, y a los que violan los parajes naturales para fines de lucro ilícito.

\section{Comercio, industria y negocios sotenibles}

Consiste en efectuar un análisis de la situación actual por la que está afrontando la industria en el país, porque de allí deriva el comercio,y se generan negocios.

a. Fortalezas

- Existencia de riquezas naturales que se pueden exportar mediante un valor agregado.

- Existencia de 85 microclimas en el Perú que favorecen el desarrollo de una gran variedad de recursos naturales.

- Posición geográfica favorable del Perú en la cuenca del Pacífico.

b. Debilidades

- Política exportadora inadecuada para las pequeñas empresas.

- Limitada promoción de recurso naturales de nuestro país.

- Existencia de maquinarias y equipos obsoletos.

c. Recomendaciones generales

- Fortalecer los Centros de Innovación Tecnológica (CITE) creados en el país.

- Promover los productos naturales con valor agregado en Ferias Internacionales.

\section{Protección y fomento de la salud}

Promover la salud humana basándose con productos alimenticios netamente de la zona o medio circundante; de esta manera, se reducirían los costos y mejoraría la nutrición, ya que los alimentos oriundos del perú tienen muchas proteínas y vitaminas.

Se debe practicar el lema "que tu alimento sea tu medicina", valorando los contenidos proteícos y vitamínicos de algunas raíces y tubérculos (maca, yacón, oca, etc.).

a. Fortalezas

- Abundancia de productos de alto valor nutritivo para consumo humano. 
- Existencia de contenidos e ingredientes activos para la salud en plantas nativas.

b. Debilidades

- Mala distribución del presupuesto destinado a la prevención y promoción de la salud.

- Desconocimiento de la relación que existe entre salud, producción de alimentos y conservación del medio ambiente.

- Deficiencia en el cumplimiento de las normas ambientales.

- El estilo de vida inadecuado para proteger y fomentar la salud humana.

- Falta de control de calidad sanitaria de los alimentos.

c. Recomendaciones generales

- Difundir a través de organismos de salud pública una información detallada a cerca de los productos que atenten contra la salud y el medio ambiente.

- Elevar el 5\% del presupuesto nacional destinado a prevención de salud.

- Exigir a las instituciones pertinentes (Ministerio de Salud, municipalidades, etc.) que cumplan los reglamentos ambientales.

- Fortalecer el sistema de control de los desechos químicos producidos por fábricas y empresas mineras.

- Crear un programa nacional de concientización a favor de la protección de la tierra en todos los organismos públicos y privados.

- Enfatizar el aspecto legislativo a fin de que las empresas y fábricas cumplan los requerimientos mínimos de control de contaminación.

\section{Turismo sostenible}

El Estado debe realizar inventarios y censos regionales de los recuros naturales al momento de entregar los lugares a las Concesiones para que devuelvan lo que explotaron: "beneficio otorgado".

El Estado debe promulgar leyes claras, coherentes con un ordenamiento ambiental completo.

La Comisión Nacional de Medio Ambiente (CONAM) debe abrir un proceso de diálogo y consulta con la sociedad civil, comunidades nativas, campesinas, etc. sobre las prioridades que deben tomarse en cuenta para procesar una eficiente legislación ambiental.

Organizar congruencia de intelectuales de diferentes especialidades para que ellos aporten en la elaboración de planes y programas sobre ecoturismo.

Establecer una adecuada organización dentro de su comunidad entre organización civil, gobiernos locales y empresa privada que permitan crear un turismo atractivo y sostenible mediante mecanis- mos de participación ciudadana para desarrollar el turismo sostenible.

a. Fortalezas

- Existencia de circuitos turísticos, monumentos arqueológicos, folclore y comidas típicas para desarrollar estudios de viabilidad del turismo.

- Incremento de canales de comunicación, teléfonos, internet en lugares alejados del país.

- Tendencia positiva a mejorar la calidad de los servicios de la infraestructura hotelera existente.

b. Debilidades

- Carencia de un plan de desarrollo regional del turismo.

- Informalidad en las empresas de servicios para la atención de turistas

- Desorden vial urbano y falta de terminales terrestres.

- Carencia de adecuado marketing turístico.

c. Recomendaciones generales

- Fomentar el turismo empezando por casa, generando conciencia turística.

- Conocer nuestros recursos que poseemos, creando tarifas especiales.

- Registros de los recursos turísticos por el Instituto Nacional de Cultura (INC) existentes y aquellos que no se conocen, pues debido a la expansión urbana se va perdiendo.

- Impulsar las coordinaciones con el INC, Ministerio de Educación, MITINCI, para generar el plan de fomento al turismo.

- Preservar aspectos típicos, costumbres culturales, impulsar la creación de circuitos gastronómicos.

\section{POLÍTICA CIENTÍFICA Y LOS GOBIERNOS REGIONALES}

Está claro que, para que una Región salga del subdesarrollo deberá impulsar y fortalecer su industria manufacturera, valorizar sus recursos naturales, humanos entre otros dando valor agregado a los productos primarios que son las materias primas. Para esto, es de mucha importancia, el diálogo continuo con los sectores diversos que se encuentran operativas, es posible el desarrollo sostenible de las regiones en el Perú, cuyo reto únicamente depende de unas pocas variables endógenas y exógenos que son factibles de manejar una Región tal como se describe en este artículo.

Una de las estrategias que deben implementar los Gobiernos Regionales que se inclinan por implantar este modelo de desarrollo sostenible regional, se refiere a las variables microeconómicas y macroeconómicas que deberán conjugar con el patrimonio de la Región y finalmente declarar como 
tesoro y reserva nacional homologados en las bolsas de valores internacionales en una alianza estratégica con los países con las cuales hará negocios y de esta manera le será relativamente fácil despegar al desarrollo regional y posteriormente al desarrollo del País.

\section{RESULTADOS ESPERADOS EN LA APLICACIÓN DEL MODELO DE DESARROLLO SOSTENIBLE REGIONAL}

Cada una de las partes del modelo de desarrollo sostenible regional que se ha descrito en esta propuesta regional, tiene sus propias alternativas de solución de acuerdo a los distintos casos específi$\cos$, los mismos que se pueden extrapolar cuando un Gobierno Regional lo ponga en marcha. Para esto se propone crear cinco áreas de trabajo:

- Investigación y desarrollo tecnológico industrial.

- Fomento para el financiamiento externo.

- Valorización de los recursos naturales de la Región (Tesoro Regional).

- Planificación de territorio regional.

- Comunicación y atención a la población menos favorecida.

Los resultados de la aplicación del modelo de desarrollo sostenible regional permitirá principalmente la:

- Mejora en la gestión total regional.

- Mejora en la calidad de vida del poblador de la Región.

- Crecimiento económico industrial en la Región.

- Incremento del valor agregado de nuestros productos regionales

- Búsqueda de mercados externos.

- Competitividad de los productos en el mercado nacional.

- Fomento a la economía sostenible.

- Mejora en los estándares de vida por incremento del PBI Regional.

- Disminución de desempleados.

\section{CONCLUSIONES}

El trinomio Gobierno Regional, Empresa y Universidad deben estar mejor consolidados, hoy más que nunca, pues de esto dependerá el funcionamiento de cualquier modelo de gestión regional a implementarse en adelante, pues hasta ahora se ha conducido al país en forma empírica y sin criterio científico, sin saber que es lo que se tiene como recursos naturales económicamente viables para el negocio estratégico con los países desarrollados, menos todavía el control económico de las mismas. Por esto nunca se hace una gestión óptima prueba de ello es que seguimos siendo subdesarrollados en más de 180 años de vida republicana.

Para determinar los factores del modelo de desarrollo sostenible se deben conformar los Focus Group y las técnicas de trabajo con participación de instituciones públicas y privadas ligados al tema, de modo que se logren identificar los problemas y las posibles soluciones a implementar.

\section{BIBLIOGRAFÍA}

1. Proyecto de Ley de Desarrollo Sostenible. 2002. Congreso de la República del Perú. Lima-Perú.

2. Declaración de Huaraz sobre Desarrollo Sostenible de los Ecosistemas de Montañas. Julio del 2002. Huaraz, Perú.

3. Declaración de Johannesburgo sobre Desarrollo Sostenible. Setiembre del 2002. Johannesburgo, South Africa.

4. OEA, Unidad de Desarrollo Sostenible y Medio Ambiente. 2001. Estrategia Interamericana para la Promoción de la Participación Pública en la Toma de Decisiones sobre Desarrollo Sostenible.USA.

5. Declaración de Rio. 1992. Agenda 21. Rio de Janerio, Brasil.

6. Druker, Peter. 1992. Managing for the future: the 1990's and Beyond. USA. 